\title{
Existence of solution to a class of boundary value problem for impulsive fractional differential equations
}

Wen-Xue Zhou ${ }^{1,2^{*}}$ and Xu Liu'

\section{*Correspondence:}

wxzhou2006@126.com

${ }^{1}$ College of Mathematics and

Physics, Lanzhou Jiaotong

University, Lanzhou, 730070, China

${ }^{2}$ School of Mathematical Sciences,

Fudan University, Shanghai, 200433, China

\begin{abstract}
By means of the Green function, the boundary value problem of a fractional differential equation can be reduced to the equivalent integral equation. Recently, this method has been used successfully to discuss the existence of the solution to the boundary value problem of a nonlinear fractional differential equation. By applying the nonlinear alternative of the Leray-Schauder type and the Krasnoselskii fixed point theorem, we investigate the boundary value problem of a nonlinear impulsive fractional differential equation, and we obtain two existence results for the solution.
\end{abstract} MSC: $26 \mathrm{~A} 33 ; 34 \mathrm{~B} 15$

Keywords: boundary value problem; impulsive fractional differential equations; Caputo fractional derivative; existence of solutions; fixed point theorem

\section{Introduction}

Boundary value problems for nonlinear fractional differential equations have recently been addressed by several researchers. The interest in the study of differential equations of fractional order lies in the fact that fractional derivatives provide an excellent tool for the description of memory and hereditary properties of various materials and processes [1-5]. For some recent development on the topic, see [6-11] and the references therein.

Impulsive differential equations, which provide a natural description of observed evolution processes, are regarded as important mathematical tools for the better understanding of several real world problems in the applied sciences. The theory of impulsive differential equations of integer order has found extensive applications in realistic mathematical modeling of a wide variety of practical situations and has emerged as an important area of investigation in recent years. For the general theory and applications of impulsive differential equations, we refer the reader to references [12-15]. The impulsive differential equations of fractional order have also attracted considerable attention and a variety of results can be found in [16-24] and the references therein.

In [16], Ahmad et al. considered the following the impulsive fractional differential equations:

$$
\left\{\begin{array}{l}
{ }^{c} D^{q} x(t)=f(t, x(t)), \quad t \in J=[0,1] \backslash\left\{t_{1}, t_{2}, t_{3}, \ldots, t_{p}\right\}, \\
\Delta x\left(t_{k}\right)=I_{k}\left(x\left(t_{k}^{-}\right)\right), \quad \Delta x^{\prime}\left(t_{k}\right)=J_{k}\left(x\left(t_{k}^{-}\right)\right), \quad t_{k} \in(0,1), k=1, \ldots, p, \\
x(0)+x^{\prime}(0)=0, \quad x(1)+x^{\prime}(1)=0,
\end{array}\right.
$$

O2014 Zhou and Liu; licensee Springer. This is an Open Access article distributed under the terms of the Creative Commons Attribution License (http://creativecommons.org/licenses/by/2.0), which permits unrestricted use, distribution, and reproduction in any medium, provided the original work is properly cited. 
where ${ }^{c} D^{q}$ is the Caputo fractional derivative. The results are based on the contraction mapping principle and Krasnoselskii's fixed point theorem.

In [17], Tian et al. considered the following the impulsive fractional differential equations:

$$
\left\{\begin{array}{l}
{ }^{c} D^{q} u(t)=f(t, u), \quad 0<t<1, t \neq t_{k}, k=1, \ldots, p, 1<q \leq 2, \\
\Delta u\left(t_{k}\right)=I_{k}\left(u\left(t_{k}^{-}\right)\right), \quad \Delta u^{\prime}\left(t_{k}\right)=\bar{I}_{k}\left(u\left(t_{k}^{-}\right)\right), \quad k=1, \ldots, p, \\
u(0)+u^{\prime}(0)=0, \quad u(1)+u^{\prime}(\xi)=0, \quad \xi \in(0,1), \xi \neq t_{k}, k=1, \ldots, p .
\end{array}\right.
$$

The results are based on the contraction mapping principle and Schauder's fixed point theorem.

In [18], Zhang et al. considered the following impulsive fractional differential equations:

$$
\left\{\begin{array}{l}
{ }^{c} D^{q} y(t)=f(t, y), \quad \forall t \in J=[0, T], t \neq t_{k}, k=1, \ldots, m, 1<q \leq 2, \\
\Delta u\left(t_{k}\right)=I_{k}\left(u\left(t_{k}^{-}\right)\right), \quad \Delta u^{\prime}\left(t_{k}\right)=\bar{I}_{k}\left(u\left(t_{k}^{-}\right)\right), \quad k=1, \ldots, p, \\
y(0)=-y(T), \quad y^{\prime}(0)=-y^{\prime}(T) .
\end{array}\right.
$$

The results are based on the Altman fixed point theorem and Leray-Schauder fixed point theorem.

On the other hand, the impulsive boundary value problems for nonlinear fractional differential equations have not been addressed so extensively and many aspects of these problems are yet to be explored. For example, we observed that in the above-mentioned work [16-24], the authors all require that the nonlinear term $f$ is bounded and continuous; if the impulse functions $I_{k}$ and $\bar{I}_{k}$ are bounded, it is easy to see that these conditions are very strongly restrictive and difficult to satisfy in applications. Motivated by the abovementioned work [16-24], this article is mainly concerned with the existence of a solution for the boundary value problems for the nonlinear impulsive fractional differential equations

$$
\left\{\begin{array}{l}
{ }^{c} D^{q} u(t)=f(t, u(t)), \quad 1<q \leq 2, t \in J^{\prime}, \\
\Delta u\left(t_{k}\right)=I_{k}\left(u\left(t_{k}^{-}\right)\right), \quad \Delta u^{\prime}\left(t_{k}\right)=\bar{I}_{k}\left(u\left(t_{k}^{-}\right)\right), \quad k=1, \ldots, m, \\
a u(0)-b u^{\prime}(0)=x_{0}, \quad c u(1)+d u^{\prime}(1)=x_{1},
\end{array}\right.
$$

where ${ }^{c} D^{q}$ is the Caputo fractional derivative, $a \geq 0, b>0, c \geq 0, d>0, \delta=a c+a d+$ $b c \neq 0$, and $x_{0}, x_{1} \in \mathbb{R} . f \in C(I \times \mathbb{R}, \mathbb{R}), I_{k}, \bar{I}_{k}: \in C(\mathbb{R}, \mathbb{R}), J=[0,1], 0=t_{0}<t_{1}<\cdots<t_{m}<$ $t_{m+1}=1, J^{\prime}=J \backslash\left\{t_{1}, t_{2}, \ldots, t_{m}\right\}, \Delta u\left(t_{k}\right)=u\left(t_{k}^{+}\right)-u\left(t_{k}^{-}\right), u\left(t_{k}^{+}\right)=\lim _{h \rightarrow 0^{+}} u\left(t_{k}+h\right)$ and $u\left(t_{k}^{-}\right)=$ $\lim _{h \rightarrow 0^{-}} u\left(t_{k}+h\right)$ represent the right and left limits of $u(t)$ at $t=t_{k}, k=1, \ldots, m . \Delta u^{\prime}\left(t_{k}\right)$ has a similar meaning for $u^{\prime}(t)$.

Evidently, problem (1.1) not only includes the boundary value problems mentioned above [16] but also extends them to a much wider case. Our main tools are the nonlinear alternative of Leray-Schauder type and the Krasnoselskii fixed point theorem. Some recent results in [16-24] are generalized and significantly improved (see Remark 3.1).

The remainder of this article is organized as follows. In Section 2, we provide some basic definitions, preliminaries facts, and various lemmas which will be used throughout this paper. In Section 3, we give two main results of problem (1.1). The last section is devoted to an example illustrating the applicability of the imposed conditions. 


\section{Preliminaries and lemmas}

Let $J_{0}=\left[0, t_{1}\right], J_{1}=\left(t_{1}, t_{2}\right], \ldots, J_{p-1}=\left(t_{p-1}, t_{p}\right], J_{p}=\left(t_{p}, 1\right]$, and let us introduce the spaces: $L^{1}(J, \mathbb{R})$ denotes the Banach space of measurable functions $u: J \mapsto \mathbb{R}$ which are Bochner integrable, equipped with the norm $\|u\|_{L^{1}}:=\int_{J}\|u(t)\| d t ; P C(J, \mathbb{R})=\{u: J \rightarrow \mathbb{R}: u \in$ $C\left(J_{k}\right), k=0,1, \ldots, m$, and $u\left(t_{k}^{+}\right)$exists, $\left.k=1, \ldots, m\right\}$ is a Banach space with the norm $\|u\|_{P C}:=$ $\sup _{t \in J}\|u(t)\|$, and $P C^{1}(J, \mathbb{R})=\left\{u: J \rightarrow \mathbb{R}: u \in C^{1}\left(J_{k}\right), k=0,1, \ldots, m\right.$, and $u\left(t_{k}^{+}\right), u^{\prime}\left(t_{k}^{+}\right)$exists, $k=1, \ldots, m\}$ is a Banach space with the norm $\|u\|_{P C^{1}}:=\max _{t \in J}\left\{\|u\|,\left\|u^{\prime}\right\|\right\}$.

Definition 2.1 [1] The Riemann-Liouville fractional integral of order $r$ for a continuous function $h$ is defined as

$$
I^{r} h(t)=\int_{0}^{t} \frac{(t-s)^{r-1}}{\Gamma(r)} h(s) d s, \quad r>0,
$$

provided the integral exists.

Definition 2.2 [1] For an at least $n$-times continuously differentiable function $h:[0$, $\infty) \rightarrow R$, the Caputo derivative of fractional order $r$ is defined as

$$
{ }^{c} D^{r} h(t)=\frac{1}{\Gamma(n-r)} \int_{0}^{t}(t-s)^{n-r-1} h^{(n)}(s) d s, \quad n-1<r<n, n=[r]+1,
$$

where $[r]$ denotes the integer part of the real number $r$.

Lemma 2.1 Let $r>0, h \in C[0,1] \cap L(0,1)$, then the differential equation ${ }^{c} D^{r} h(t)=0$ has solutions

$$
h(t)=c_{0}+c_{1} t+c_{2} t^{2}+\cdots+c_{n-1} t^{n-1},
$$

where $c_{i} \in \mathbb{R}, i=0,1,2, \ldots, n-1, n=[r]+1$.

Lemma 2.2 Assume that $h \in C[0,1] \cap L(0,1)$ with a derivative of order $r$ that belongs to $C[0,1] \cap L(0,1)$. Then

$$
I_{0+}^{r}{ }^{c} D_{0+}^{r} h(t)=h(t)+c_{0}+c_{1} t+c_{2} t^{2}+\cdots+c_{n-1} t^{n-1},
$$

where $c_{i} \in \mathbb{R}, i=0,1,2, \ldots, n-1, n=[r]+1$.

Lemma 2.3 [22] For a given $h \in C[0,1]$, a function $u$ is a solution of the following impulsive boundary value problem:

$$
\left\{\begin{array}{l}
{ }^{c} D^{q} u(t)=h(t), \quad 1<q \leq 2, t \in J^{\prime}, \\
\Delta u\left(t_{k}\right)=I_{k}\left(u\left(t_{k}^{-}\right)\right), \quad \Delta u^{\prime}\left(t_{k}\right)=\bar{I}_{k}\left(u\left(t_{k}^{-}\right)\right), \quad k=1, \ldots, m, \\
a u(0)-b u^{\prime}(0)=x_{0}, \quad c u(1)+d u^{\prime}(1)=x_{1},
\end{array}\right.
$$


if and only if $u$ is a solution of the impulsive fractional integral equation

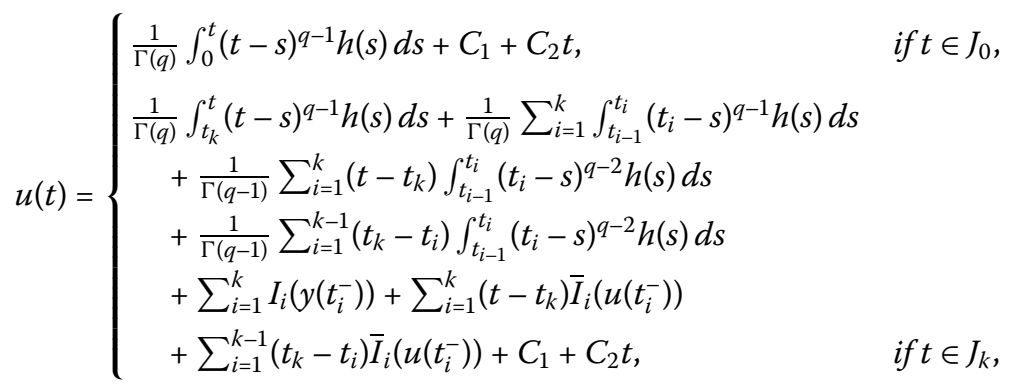

where

$$
\begin{aligned}
C_{1}= & -\left\{\sum_{i=1}^{m+1} \frac{b c}{\delta \Gamma(q)} \int_{t_{i-1}}^{t_{i}}\left(t_{i}-s\right)^{q-1} h(s) d s+\sum_{i=1}^{m} \frac{b c\left(1-t_{m}\right)}{\delta \Gamma(q-1)} \int_{t_{i-1}}^{t_{i}}\left(t_{i}-s\right)^{q-2} h(s) d s\right. \\
& +\sum_{i=1}^{m-1} \frac{b c\left(t_{m}-t_{i}\right)}{\delta \Gamma(q-1)} \int_{t_{i-1}}^{t_{i}}\left(t_{i}-s\right)^{q-2} h(s) d s+\sum_{i=1}^{m+1} \frac{b d}{\delta \Gamma(q-1)} \int_{t_{i-1}}^{t_{i}}\left(t_{i}-s\right)^{q-2} h(s) d s \\
& +\sum_{i=1}^{m} \frac{b c}{\delta} I_{i}\left(u\left(t_{i}^{-}\right)\right)+\sum_{i=1}^{m} \frac{b c\left(1-t_{p}\right)}{\delta} \bar{I}_{i}\left(u\left(t_{i}^{-}\right)\right)+\sum_{i=1}^{m-1} \frac{b c\left(t_{p}-t_{i}\right)}{\delta} \bar{I}_{i}\left(u\left(t_{i}^{-}\right)\right) \\
& \left.+\sum_{i=1}^{m} \frac{b d}{\delta} \bar{I}_{i}\left(u\left(t_{i}^{-}\right)\right)+\frac{(b c-\delta) x_{0}-a b x_{1}}{a \delta}\right\}, \\
C_{2}= & -\left\{\sum_{i=1}^{m+1} \frac{a c}{\delta \Gamma(q)} \int_{t_{i-1}}^{t_{i}}\left(t_{i}-s\right)^{q-1} h(s) d s+\sum_{i=1}^{m} \frac{a c\left(1-t_{m}\right)}{\delta \Gamma(q-1)} \int_{t_{i-1}}^{t_{i}}\left(t_{i}-s\right)^{q-2} h(s) d s\right. \\
& +\sum_{i=1}^{m-1} \frac{a c\left(t_{m}-t_{i}\right)}{\delta \Gamma(q-1)} \int_{t_{i-1}}^{t_{i}}\left(t_{i}-s\right)^{q-2} h(s) d s+\sum_{i=1}^{m+1} \frac{a d}{\delta \Gamma(q-1)} \int_{t_{i-1}}^{t_{i}}\left(t_{i}-s\right)^{q-2} h(s) d s \\
& +\sum_{i=1}^{m} \frac{a c}{\delta} I_{i}\left(u\left(t_{i}^{-}\right)\right)+\sum_{i=1}^{m} \frac{a c\left(1-t_{p}\right)}{\delta} \bar{I}_{i}\left(u\left(t_{i}^{-}\right)\right)+\sum_{i=1}^{m-1} \frac{a c\left(t_{p}-t_{i}\right)}{\delta} \bar{I}_{i}\left(u\left(t_{i}^{-}\right)\right) \\
& \left.+\sum_{i=1}^{m} \frac{a d}{\delta} \bar{I}_{i}\left(u\left(t_{i}^{-}\right)\right)+\frac{c x_{0}-a x_{1}}{a \delta}\right\} .
\end{aligned}
$$

Now we state some well-known fixed point theorems which are needed to prove the existence of solutions for equation (1.1).

Lemma 2.4 [25] (the nonlinear alternative of Leray-Schauder) Let E be a Banach space, $C$ a convex subset of $E, U$ an open subset of $C$ and $0 \in U$. Suppose $F: \bar{U} \rightarrow C$ (here $\bar{U}$ denotes the closure of $U$ in $C)$ is a continuous, compact map. Then either

(A1) $F$ has a fixed point in $U$; or

(A2) there exists $u \in \partial U$ (the boundary of $U$ in $C$ ) and $\lambda \in(0,1)$ with $u=\lambda F(u)$.

Lemma 2.5 [25] (Krasnoselskii fixed point theorem) Let $\mathcal{D}$ be a closed convex and nonempty subset of a Banach space X. Let $\mathcal{A}_{1}, \mathcal{A}_{2}$ be the operators such that

(i) $\mathcal{A}_{1} x+\mathcal{A}_{2} y \in \mathcal{D}$ whenever $x, y \in \mathcal{D}$;

(ii) $\mathcal{A}_{1}$ is completely continuous; 
(iii) $\mathcal{A}_{2}$ is a contraction mapping.

Then there exists $z \in \mathcal{D}$ such that $z=\mathcal{A}_{1} z+\mathcal{A}_{2} z$.

\section{Main results}

Define an operator $\mathcal{A}: P C(J, \mathbb{R}) \rightarrow P C(J, \mathbb{R})$ as

$$
\begin{aligned}
(\mathcal{A} u)(t)= & \frac{1}{\Gamma(q)} \int_{t_{k}}^{t}(t-s)^{q-1} f(s, u(s)) d s+\frac{1}{\Gamma(q)} \sum_{i=1}^{k} \int_{t_{i-1}}^{t_{i}}\left(t_{i}-s\right)^{q-1} f(s, u(s)) d s \\
& +\frac{1}{\Gamma(q-1)} \sum_{i=1}^{k}\left(t-t_{k}\right) \int_{t_{i-1}}^{t_{i}}\left(t_{i}-s\right)^{q-2} f(s, u(s)) d s \\
& +\frac{1}{\Gamma(q-1)} \sum_{i=1}^{k-1}\left(t_{k}-t_{i}\right) \int_{t_{i-1}}^{t_{i}}\left(t_{i}-s\right)^{q-2} f(s, u(s)) d s+\sum_{i=1}^{k} I_{i}\left(u\left(t_{i}^{-}\right)\right) \\
& +\sum_{i=1}^{k}\left(t-t_{k}\right) \bar{I}_{i}\left(u\left(t_{i}^{-}\right)\right)+\sum_{i=1}^{k-1}\left(t_{k}-t_{i}\right) \bar{I}_{i}\left(u\left(t_{i}^{-}\right)\right)+M_{1}+M_{2} t,
\end{aligned}
$$

where

$$
\begin{aligned}
M_{1}= & -\left\{\sum_{i=1}^{m+1} \int_{t_{i-1}}^{t_{i}} \frac{b c\left(t_{i}-s\right)^{q-1}}{\delta \Gamma(q)} f(s, u(s)) d s+\sum_{i=1}^{m} \int_{t_{i-1}}^{t_{i}} \frac{b c\left(1-t_{m}\right)\left(t_{i}-s\right)^{q-2}}{\delta \Gamma(q-1)} f(s, u(s)) d s\right. \\
& +\sum_{i=1}^{m-1} \int_{t_{i-1}}^{t_{i}} \frac{b c\left(t_{m}-t_{i}\right)\left(t_{i}-s\right)^{q-2}}{\delta \Gamma(q-1)} f(s, u(s)) d s+\sum_{i=1}^{m+1} \int_{t_{i-1}}^{t_{i}} \frac{b d\left(t_{i}-s\right)^{q-2}}{\delta \Gamma(q-1)} f(s, u(s)) d s \\
& +\sum_{i=1}^{m} \frac{b c}{\delta} I_{i}\left(u\left(t_{i}^{-}\right)\right)+\sum_{i=1}^{m} \frac{b c\left(1-t_{p}\right)}{\delta} \bar{I}_{i}\left(u\left(t_{i}^{-}\right)\right)+\sum_{i=1}^{m-1} \frac{b c\left(t_{p}-t_{i}\right)}{\delta} \bar{I}_{i}\left(u\left(t_{i}^{-}\right)\right) \\
& \left.+\sum_{i=1}^{m} \frac{b d}{\delta} \bar{I}_{i}\left(u\left(t_{i}^{-}\right)\right)+\frac{(b c-\delta) x_{0}-a b x_{1}}{a \delta}\right\}, \\
M_{2}= & -\left\{\sum_{i=1}^{m+1} \int_{t_{i-1}}^{t_{i}} \frac{a c\left(t_{i}-s\right)^{q-1}}{\delta \Gamma(q)} f(s, u(s)) d s+\sum_{i=1}^{m} \int_{t_{i-1}}^{t_{i}} \frac{a c\left(1-t_{m}\right)\left(t_{i}-s\right)^{q-2}}{\delta \Gamma(q-1)} f(s, u(s)) d s\right. \\
& +\sum_{i=1}^{m-1} \int_{t_{i-1}}^{t_{i}} \frac{a c\left(t_{m}-t_{i}\right)\left(t_{i}-s\right)^{q-2}}{\delta \Gamma(q-1)} f(s, u(s)) d s+\sum_{i=1}^{m+1} \int_{t_{i-1}}^{t_{i}} \frac{a d\left(t_{i}-s\right)^{q-2}}{\delta \Gamma(q-1)} f(s, u(s)) d s \\
& +\sum_{i=1}^{m} \frac{a c}{\delta} I_{i}\left(u\left(t_{i}^{-}\right)\right)+\sum_{i=1}^{m} \frac{a c\left(1-t_{p}\right)}{\delta} \bar{I}_{i}\left(u\left(t_{i}^{-}\right)\right)+\sum_{i=1}^{m-1} \frac{b c\left(t_{p}-t_{i}\right)}{\delta} \bar{I}_{i}\left(u\left(t_{i}^{-}\right)\right) \\
& \left.+\sum_{i=1}^{m} \frac{a d}{\delta} \bar{I}_{i}\left(u\left(t_{i}^{-}\right)\right)+\frac{c x_{0}-a x_{1}}{a \delta}\right\} .
\end{aligned}
$$

Lemma 3.1 [22] Let $f \in C(I \times \mathbb{R}, \mathbb{R}), I_{k}, \bar{I}_{k}: C(\mathbb{R}, \mathbb{R})$, then $\mathcal{A}: P C(J, \mathbb{R}) \rightarrow P C(J, \mathbb{R})$ is completely continuous.

Theorem 3.1 Assume that the following conditions hold.

(H1) The function $f \in C(I \times \mathbb{R}, \mathbb{R}), I_{k}, \bar{I}_{k}: \mathbb{R} \rightarrow \mathbb{R}$ is continuous. 
(H2) There exist $\phi_{f} \in C(J,[0,+\infty))$ and $\varphi:[0,+\infty) \rightarrow[0,+\infty)$ continuous and nondecreasing such that

$$
|f(t, u)| \leq \phi_{f}(t) \varphi(|u|), \quad(t, u) \in J \times \mathbb{R} .
$$

(H3) There exist $\varphi^{\star}, \bar{\varphi}^{\star}:[0,+\infty) \rightarrow[0,+\infty)$ continuous and nondecreasing such that

$$
\left|I_{k}(u)\right| \leq \varphi^{\star}(|u|), \quad\left|\bar{I}_{k}(u)\right| \leq \bar{\varphi}^{\star}(|u|), \quad u \in \mathbb{R} .
$$

(H4) There exists a number $\bar{M}>0$ such that

$$
\frac{\bar{M}}{A \phi_{f}^{0} \varphi(\bar{M})+B \varphi^{\star}(\bar{M})+C \bar{\varphi}^{\star}(\bar{M})+D}>1,
$$

where

$$
\begin{aligned}
& A=\frac{(m+1)[c(a+b)+\delta]}{\delta \Gamma(q+1)}+\frac{(2 m-1)[c(a+b)+\delta]+(m+1)(a+b) d}{\delta \Gamma(q)}, \\
& B=\frac{m[c(a+b)+\delta]}{\delta}, \\
& C=\frac{(2 m-1)[c(a+b)+\delta]+m d(a+b)}{\delta}, \quad D=\frac{(2 c+d)\left|x_{0}\right|+(a+b)\left|x_{1}\right|}{\delta} .
\end{aligned}
$$

Then problem (1.1) has at least one solution on J.

Proof Consider the operator $\mathcal{A}$ defined by (3.1). By Lemma 3.1, it can easily be shown that $\mathcal{A}$ is continuous and completely continuous. For $0 \leq \lambda \leq 1$, let $u$ be such that for each $t \in J$ we have $u(t)=\lambda(\mathcal{A} u)(t)$. Then from (H2)-(H3) we have for each $t \in J$,

$$
\begin{aligned}
\left|M_{1}\right| \leq & \sum_{i=1}^{m+1} \int_{t_{i-1}}^{t_{i}} \frac{b c\left(t_{i}-s\right)^{q-1}}{\delta \Gamma(q)}|f(s, u(s))| d s+\sum_{i=1}^{m} \int_{t_{i-1}}^{t_{i}} \frac{b c\left(1-t_{m}\right)\left(t_{i}-s\right)^{q-2}}{\delta \Gamma(q-1)}|f(s, u(s))| d s \\
& +\sum_{i=1}^{m-1} \int_{t_{i-1}}^{t_{i}} \frac{b c\left(t_{m}-t_{i}\right)\left(t_{i}-s\right)^{q-2}}{\delta \Gamma(q-1)}|f(s, u(s))| d s \\
& +\sum_{i=1}^{m+1} \int_{t_{i-1}}^{t_{i}} \frac{b d\left(t_{i}-s\right)^{q-2}}{\delta \Gamma(q-1)}|f(s, u(s))| d s \\
& +\sum_{i=1}^{m} \frac{b c}{\delta}\left|I_{i}\left(u\left(t_{i}\right)\right)\right|+\sum_{i=1}^{m} \frac{b c\left(1-t_{p}\right)}{\delta}\left|\bar{I}_{i}\left(u\left(t_{i}\right)\right)\right|+\sum_{i=1}^{m-1} \frac{b c\left(t_{p}-t_{i}\right)}{\delta}\left|\bar{I}_{i}\left(u\left(t_{i}\right)\right)\right| \\
& +\sum_{i=1}^{m} \frac{b d}{\delta}\left|\bar{I}_{i}\left(u\left(t_{i}\right)\right)\right|+\frac{(c+d)\left|x_{0}\right|+b\left|x_{1}\right|}{\delta} \\
\leq & \sum_{i=1}^{m+1} \int_{t_{i-1}}^{t_{i}} \frac{b c\left(t_{i}-s\right)^{q-1}}{\delta \Gamma(q)} \phi_{f}(s) \varphi(|u(s)|) d s+\sum_{i=1}^{m} \frac{b c}{\delta} \varphi^{\star}\left(\left|u\left(t_{i}\right)\right|\right) \\
& +\sum_{i=1}^{m} \int_{t_{i-1}}^{t_{i}} \frac{b c\left(1-t_{m}\right)\left(t_{i}-s\right)^{q-2}}{\delta \Gamma(q-1)} \phi_{f}(s) \varphi(|u(s)|) d s+\sum_{i=1}^{m} \frac{b c\left(1-t_{p}\right)}{\delta} \bar{\varphi}^{\star}\left(\left|u\left(t_{i}\right)\right|\right)
\end{aligned}
$$




$$
\begin{aligned}
& +\sum_{i=1}^{m-1} \int_{t_{i-1}}^{t_{i}} \frac{b c\left(t_{m}-t_{i}\right)\left(t_{i}-s\right)^{q-2}}{\delta \Gamma(q-1)} \phi_{f}(s) \varphi(|u(s)|) d s+\sum_{i=1}^{m-1} \frac{b c\left(t_{p}-t_{i}\right)}{\delta} \bar{\varphi}^{\star}\left(\left|u\left(t_{i}\right)\right|\right) \\
& +\sum_{i=1}^{m+1} \int_{t_{i-1}}^{t_{i}} \frac{b d\left(t_{i}-s\right)^{q-2}}{\delta \Gamma(q-1)} \phi_{f}(s) \varphi(|u(s)|) d s+\sum_{i=1}^{m} \frac{b d}{\delta} \bar{\varphi}^{\star}\left(\left|u\left(t_{i}\right)\right|\right) \\
& +\frac{(c+d)\left|x_{0}\right|+b\left|x_{1}\right|}{\delta} \\
& \leq\left[\frac{(m+1) b c}{\delta \Gamma(q+1)}+\frac{m b c}{\delta \Gamma(q)}+\frac{(m-1) b c}{\delta \Gamma(q)}+\frac{(m+1) b d}{\delta \Gamma(q)}\right] \phi_{f}^{0} \varphi\left(\|u\|_{\infty}\right)+\frac{m b c}{\delta} \varphi^{\star}\left(\|u\|_{\infty}\right) \\
& +\left[\frac{m b c}{\delta}+\frac{(m-1) b c}{\delta}+\frac{m b d}{\delta}\right] \bar{\varphi}^{\star}\left(\|u\|_{\infty}\right)+\frac{(c+d)\left|x_{0}\right|+b\left|x_{1}\right|}{\delta}
\end{aligned}
$$

Similarly, we have

$$
\begin{aligned}
\left|M_{2}\right| \leq & \sum_{i=1}^{m+1} \int_{t_{i-1}}^{t_{i}} \frac{a c\left(t_{i}-s\right)^{q-1}}{\delta \Gamma(q)} \phi_{f}(s) \varphi(|u(s)|) d s+\sum_{i=1}^{m} \frac{b c}{\delta} \varphi^{\star}\left(\left|u\left(t_{i}\right)\right|\right) \\
& +\sum_{i=1}^{m} \int_{t_{i-1}}^{t_{i}} \frac{a c\left(1-t_{m}\right)\left(t_{i}-s\right)^{q-2}}{\delta \Gamma(q-1)} \phi_{f}(s) \varphi(|u(s)|) d s+\sum_{i=1}^{m} \frac{a c\left(1-t_{p}\right)}{\delta} \bar{\varphi}^{\star}\left(\left|u\left(t_{i}\right)\right|\right) \\
& +\sum_{i=1}^{m-1} \int_{t_{i-1}}^{t_{i}} \frac{a c\left(t_{m}-t_{i}\right)\left(t_{i}-s\right)^{q-2}}{\delta \Gamma(q-1)} \phi_{f}(s) \varphi(|u(s)|) d s+\sum_{i=1}^{m-1} \frac{a c\left(t_{p}-t_{i}\right)}{\delta} \bar{\varphi}^{\star}\left(\left|u\left(t_{i}\right)\right|\right) \\
& +\sum_{i=1}^{m+1} \int_{t_{i-1}}^{t_{i}} \frac{a d\left(t_{i}-s\right)^{q-2}}{\delta \Gamma(q-1)} \phi_{f}(s) \varphi(|u(s)|) d s+\sum_{i=1}^{m} \frac{a d}{\delta} \bar{\varphi}^{\star}\left(\left|u\left(t_{i}\right)\right|\right)+\frac{c\left|x_{0}\right|+a\left|x_{1}\right|}{\delta} \\
\leq & {\left[\frac{(m+1) a c}{\delta \Gamma(q+1)}+\frac{m a c}{\delta \Gamma(q)}+\frac{(m-1) a c}{\delta \Gamma(q)}+\frac{(m+1) a d}{\delta \Gamma(q)}\right] \phi_{f}^{0} \varphi\left(\|u\|_{\infty}\right)+\frac{m a c}{\delta} \varphi^{\star}\left(\|u\|_{\infty}\right) } \\
& +\left[\frac{m a c}{\delta}+\frac{(m-1) a c}{\delta}+\frac{m b d}{\delta}\right] \bar{\varphi}^{\star}\left(\|u\|_{\infty}\right)+\frac{c\left|x_{0}\right|+a\left|x_{1}\right|}{\delta}
\end{aligned}
$$

Therefore

$$
\begin{aligned}
|u(t)| \leq & \frac{1}{\Gamma(q)} \int_{t_{k}}^{t}(t-s)^{q-1}|f(s, u(s))| d s+\frac{1}{\Gamma(q)} \sum_{i=1}^{k} \int_{t_{i-1}}^{t_{i}}\left(t_{i}-s\right)^{q-1}|f(s, u(s))| d s \\
& +\frac{1}{\Gamma(q-1)} \sum_{i=1}^{k}\left(t-t_{k}\right) \int_{t_{i-1}}^{t_{i}}\left(t_{i}-s\right)^{q-2}|f(s, u(s))| d s \\
& +\frac{1}{\Gamma(q-1)} \sum_{i=1}^{k-1}\left(t_{k}-t_{i}\right) \int_{t_{i-1}}^{t_{i}}\left(t_{i}-s\right)^{q-2}|f(s, u(s))| d s+\sum_{i=1}^{k}\left|I_{i}\left(u\left(t_{i}^{-}\right)\right)\right| \\
& +\sum_{i=1}^{k}\left(t-t_{k}\right)\left|\bar{I}_{i}\left(u\left(t_{i}^{-}\right)\right)\right|+\sum_{i=1}^{k-1}\left(t_{k}-t_{i}\right)\left|\bar{I}_{i}\left(u\left(t_{i}^{-}\right)\right)\right|+\left|M_{1}\right|+\left|M_{2}\right| \\
\leq & {\left[\int_{t_{k}}^{t} \frac{(t-s)^{q-1}}{\Gamma(q)} d s+\sum_{i=1}^{m} \int_{t_{i-1}}^{t_{i}} \frac{\left(t_{i}-s\right)^{q-1}}{\Gamma(q)} d s+\left|M_{1}\right|+\left|M_{2}\right|\right.} \\
& \left.+\sum_{i=1}^{m} \int_{t_{i-1}}^{t_{i}} \frac{\left(t-t_{k}\right)\left(t_{i}-s\right)^{q-2}}{\Gamma(q-1)} d s+\sum_{i=1}^{m-1} \int_{t_{i-1}}^{t_{i}} \frac{\left(t_{k}-t_{i}\right)\left(t_{i}-s\right)^{q-2}}{\Gamma(q-1)} d s\right] \phi_{f}^{0} \varphi\left(\|u\|_{\infty}\right)
\end{aligned}
$$




$$
\begin{aligned}
& +\sum_{i=1}^{m} \varphi^{\star}\left(\left|u\left(t_{k}\right)\right|\right)+\sum_{i=1}^{m}\left(t-t_{k}\right) \bar{\varphi}^{\star}\left(\left|u\left(t_{k}\right)\right|\right)+\sum_{i=1}^{m-1}\left(t_{k}-t_{i}\right) \bar{\varphi}^{\star}\left(\left|u\left(t_{k}\right)\right|\right) \\
\leq & \frac{(m+1)[c(a+b)+\delta]}{\delta \Gamma(q+1)} \phi_{f}^{0} \varphi\left(\|u\|_{\infty}\right) \\
& +\frac{(2 m-1)[c(a+b)+\delta]+(m+1)(a+b) d}{\delta \Gamma(q)} \phi_{f}^{0} \varphi\left(\|u\|_{\infty}\right) \\
& +\frac{m[c(a+b)+\delta]}{\delta} \varphi^{\star}\left(\|u\|_{\infty}\right)+\frac{(2 m-1)[c(a+b)+\delta]+m d(a+b)}{\delta} \bar{\varphi}^{\star}\left(\|u\|_{\infty}\right) \\
& +\frac{(2 c+d)\left|x_{0}\right|+(a+b)\left|x_{1}\right|}{\delta},
\end{aligned}
$$

which implies that

$$
\frac{\|u\|_{\infty}}{A \phi_{f}^{0} \varphi\left(\|u\|_{\infty}\right)+B \varphi^{\star}\left(\|u\|_{\infty}\right)+C \bar{\varphi}^{\star}\left(\|u\|_{\infty}\right)+D} \leq 1
$$

Then by the condition (3.4) there exists $\bar{M}$ such that $\|u\|_{\infty} \neq \bar{M}$.

Let

$$
U=\left\{u \in P C(J, \mathbb{R}):\|u\|_{\infty}<\bar{M}\right\} .
$$

The operator $\mathcal{A}: \bar{U} \rightarrow P C(J, \mathbb{R})$ is continuous and completely continuous. From the choice of $U$, there is no $u \in \partial U$ such that $u=\lambda \mathcal{A}(u)$ for some $\lambda \in[0,1]$. As a consequence of the nonlinear alternative of Leray-Schauder type [25] we deduce that $\mathcal{A}$ has a fixed point $u$ in $\bar{U}$ which is a solution of problem (1.1). This completes the proof.

Remark 3.1 Compared with Theorem 3.2 in [16-22], our Theorem 3.1 does not need conditions $|f(t, u)| \leq L_{1},\left|I_{k}(u)\right| \leq L_{2},\left|\bar{I}_{k}(u)\right| \leq L_{3}$, clearly, these conditions are very strong. Thus, the results of the above-mentioned works are generalized and significantly improved.

Theorem 3.2 Let $f \in C(I \times \mathbb{R}, \mathbb{R}), I_{k}, \bar{I}_{k}: C(\mathbb{R}, \mathbb{R})$, and they satisfy

(H5) there exists a positive constant $\gamma_{1}>0$, such that

$$
|f(t, u)-f(t, v)| \leq \gamma_{1}|u-v|, \quad \forall t \in J, u, v \in \mathbb{R}
$$

(H6) there exist positive constants $\gamma_{2}, \gamma_{3}, \gamma_{4}, \gamma_{5}>0$, for $\forall u, v \in \mathbb{R}$, such that

$$
\begin{aligned}
& \left|I_{k}(u)-I_{k}(v)\right| \leq \gamma_{2}|u-v|, \quad\left|\bar{I}_{k}(u)-\bar{I}_{k}(v)\right| \leq \gamma_{3}|u-v|, \\
& \left|I_{k}(u)\right| \leq \gamma_{4}, \quad\left|\bar{I}_{k}(u)\right| \leq \gamma_{5}, \quad k=1, \ldots, m ;
\end{aligned}
$$

(H7) for $(t, x) \in J \times \mathbb{R}$ and $\mu \in P C\left(J, \mathbb{R}^{+}\right)$, one has

$$
|f(t, x(t))| \leq \mu(t)
$$


and

$$
\begin{aligned}
\lambda:= & \frac{m[c(a+b)+\delta]}{\delta} \gamma_{4}+\frac{(2 m-1)[c(a+b)+\delta]+m d(a+b)}{\delta} \gamma_{5} \\
& +\frac{(2 c+d)\left|x_{0}\right|+(a+b)\left|x_{1}\right|}{\delta}<1 .
\end{aligned}
$$

Then problem (1.1) has at least one solution.

Proof Let us fix

$$
\begin{aligned}
r \geq & \left\{\frac{(m+1)[c(a+b)+\delta]}{\delta \Gamma(q+1)}+\frac{(2 m-1)[c(a+b)+\delta]+(m+1)(a+b) d}{\delta \Gamma(q)}\right\}\|\mu\|_{P C} \\
& +\frac{m[c(a+b)+\delta]}{\delta} \gamma_{4}+\frac{(2 m-1)[c(a+b)+\delta]+m d(a+b)}{\delta} \gamma_{5} \\
& +\frac{(2 c+d)\left|x_{0}\right|+(a+b)\left|x_{1}\right|}{\delta} .
\end{aligned}
$$

Let $\mathbb{C}=P C(J, \mathbb{R})$, and consider $B_{r}=\{u \in \mathbb{C}:\|u\| \leq r\}$; then $B_{r}$ is a bounded, closed, convex set in $\mathbb{C}$.

Now define the operators $\mathcal{A}_{1}$ and $\mathcal{A}_{2}$ on $B_{r}$ as

$$
\begin{aligned}
& \left(\mathcal{A}_{1} u\right)(t) \\
& =\frac{1}{\Gamma(q)} \int_{t_{k}}^{t}(t-s)^{q-1} f(s, u(s)) d s+\frac{1}{\Gamma(q)} \sum_{i=1}^{m} \int_{t_{i-1}}^{t_{i}}\left(t_{i}-s\right)^{q-1} f(s, u(s)) d s \\
& +\frac{1}{\Gamma(q-1)} \sum_{i=1}^{m}\left(t-t_{k}\right) \int_{t_{i-1}}^{t_{i}}\left(t_{i}-s\right)^{q-2} f(s, u(s)) d s \\
& +\frac{1}{\Gamma(q-1)} \sum_{i=1}^{m-1}\left(t_{k}-t_{i}\right) \int_{t_{i-1}}^{t_{i}}\left(t_{i}-s\right)^{q-2} f(s, u(s)) d s \\
& -\left\{\sum_{i=1}^{m+1} \int_{t_{i-1}}^{t_{i}} \frac{b c\left(t_{i}-s\right)^{q-1}}{\delta \Gamma(q)} f(s, u(s)) d s+\sum_{i=1}^{m} \int_{t_{i-1}}^{t_{i}} \frac{b c\left(1-t_{m}\right)\left(t_{i}-s\right)^{q-2}}{\delta \Gamma(q-1)} f(s, u(s)) d s\right. \\
& +\sum_{i=1}^{m-1} \int_{t_{i-1}}^{t_{i}} \frac{b c\left(t_{m}-t_{i}\right)\left(t_{i}-s\right)^{q-2}}{\delta \Gamma(q-1)} f(s, u(s)) d s \\
& \left.+\sum_{i=1}^{m+1} \int_{t_{i-1}}^{t_{i}} \frac{b d\left(t_{i}-s\right)^{q-2}}{\delta \Gamma(q-1)} f(s, u(s)) d s\right\} \\
& -\left\{\sum_{i=1}^{m+1} \int_{t_{i-1}}^{t_{i}} \frac{a c\left(t_{i}-s\right)^{q-1}}{\delta \Gamma(q)} f(s, u(s)) d s+\sum_{i=1}^{m} \int_{t_{i-1}}^{t_{i}} \frac{a c\left(1-t_{m}\right)\left(t_{i}-s\right)^{q-2}}{\delta \Gamma(q-1)} f(s, u(s)) d s\right. \\
& +\sum_{i=1}^{m-1} \int_{t_{i-1}}^{t_{i}} \frac{a c\left(t_{m}-t_{i}\right)\left(t_{i}-s\right)^{q-2}}{\delta \Gamma(q-1)} f(s, u(s)) d s \\
& \left.+\sum_{i=1}^{m+1} \int_{t_{i-1}}^{t_{i}} \frac{\operatorname{ad}\left(t_{i}-s\right)^{q-2}}{\delta \Gamma(q-1)} f(s, u(s)) d s\right\} t
\end{aligned}
$$


and

$$
\begin{aligned}
\left(\mathcal{A}_{2} u\right)(t)= & \sum_{i=1}^{m} I_{i}\left(u\left(t_{i}^{-}\right)\right)+\sum_{i=1}^{m}\left(t-t_{k}\right) \bar{I}_{i}\left(u\left(t_{i}^{-}\right)\right)+\sum_{i=1}^{m-1}\left(t_{k}-t_{i}\right) \bar{I}_{i}\left(u\left(t_{i}^{-}\right)\right) \\
& -\left\{\sum_{i=1}^{m} \frac{b c}{\delta} I_{i}\left(u\left(t_{i}^{-}\right)\right)+\sum_{i=1}^{m} \frac{b c\left(1-t_{p}\right)}{\delta} \bar{I}_{i}\left(u\left(t_{i}^{-}\right)\right)\right. \\
& \left.+\sum_{i=1}^{m-1} \frac{b c\left(t_{p}-t_{i}\right)}{\delta} \bar{I}_{i}\left(u\left(t_{i}^{-}\right)\right)+\sum_{i=1}^{m} \frac{b d}{\delta} \bar{I}_{i}\left(u\left(t_{i}^{-}\right)\right)+\frac{(b c-\delta) x_{0}-a b x_{1}}{a \delta}\right\} \\
& -\left\{\sum_{i=1}^{m} \frac{a c}{\delta} I_{i}\left(u\left(t_{i}^{-}\right)\right)+\sum_{i=1}^{m} \frac{a c\left(1-t_{p}\right)}{\delta} \bar{I}_{i}\left(u\left(t_{i}^{-}\right)\right)\right. \\
& \left.+\sum_{i=1}^{m-1} \frac{b c\left(t_{p}-t_{i}\right)}{\delta} \bar{I}_{i}\left(u\left(t_{i}^{-}\right)\right)+\sum_{i=1}^{m} \frac{a d}{\delta} \bar{I}_{i}\left(u\left(t_{i}^{-}\right)\right)+\frac{c x_{0}-a x_{1}}{a \delta}\right\} t .
\end{aligned}
$$

For $u, v \in B_{r}$, by $(\mathrm{H} 7)$, we find that

$$
\begin{aligned}
\left\|\mathcal{A}_{1} u+\mathcal{A}_{2} v\right\| \\
\leq\left\{\frac{(m+1)[c(a+b)+\delta]}{\delta \Gamma(q+1)}+\frac{(2 m-1)[c(a+b)+\delta]+(m+1)(a+b) d}{\delta \Gamma(q)}\right\}\|\mu\|_{P C} \\
\quad+\frac{m[c(a+b)+\delta]}{\delta} \gamma_{4}+\frac{(2 m-1)[c(a+b)+\delta]+m d(a+b)}{\delta} \gamma_{5} \\
\quad+\frac{(2 c+d)\left|x_{0}\right|+(a+b)\left|x_{1}\right|}{\delta}
\end{aligned}
$$

$\leq r$

Thus, $\left\|\mathcal{A}_{1} u+\mathcal{A}_{2} v\right\| \leq r$, so $\mathcal{A}_{1} u+\mathcal{A}_{2} v \in B_{r}$.

For $\forall u, v \in B_{r}$ and for each $t \in J$, it follows from the assumption (H6) that $\mathcal{A}_{2}$ is a contraction mapping for $\lambda<1$. Continuity of $f$ implies that the operator $\mathcal{A}_{1}$ is continuous. Also, $\mathcal{A}_{1}$ is uniformly bounded on $B_{r}$. In fact,

$$
\begin{aligned}
\left\|\mathcal{A}_{1} x\right\| \leq & \left\{\frac{(m+1)[c(a+b)+\delta]}{\delta \Gamma(q+1)}\right. \\
& \left.+\frac{(2 m-1)[c(a+b)+\delta]+(m+1)(a+b) d}{\delta \Gamma(q)}\right\}\|\mu\|_{P C} .
\end{aligned}
$$

On the other hand, for $\forall t \in J_{k}, 0 \leq k \leq m$, we have

$$
\begin{aligned}
\left|\left(\mathcal{A}_{1} u\right)^{\prime}(t)\right| & \\
\leq & \frac{1}{\Gamma(q-1)} \int_{t_{k}}^{t}(t-s)^{q-2}|f(s, u(s))| d s+\frac{1}{\Gamma(q-1)} \sum_{i=1}^{m} \int_{t_{i-1}}^{t_{i}}\left(t_{i}-s\right)^{q-2}|f(s, u(s))| d s \\
& +\mid\left\{\sum_{i=1}^{m+1} \int_{t_{i-1}}^{t_{i}} \frac{a c\left(t_{i}-s\right)^{q-1}}{\delta \Gamma(q)} f(s, u(s)) d s+\sum_{i=1}^{m} \int_{t_{i-1}}^{t_{i}} \frac{a c\left(1-t_{m}\right)\left(t_{i}-s\right)^{q-2}}{\delta \Gamma(q-1)} f(s, u(s)) d s\right.
\end{aligned}
$$




$$
\begin{aligned}
& \left.+\sum_{i=1}^{m-1} \int_{t_{i-1}}^{t_{i}} \frac{a c\left(t_{m}-t_{i}\right)\left(t_{i}-s\right)^{q-2}}{\delta \Gamma(q-1)} f(s, u(s)) d s+\sum_{i=1}^{m+1} \int_{t_{i-1}}^{t_{i}} \frac{a d\left(t_{i}-s\right)^{q-2}}{\delta \Gamma(q-1)} f(s, u(s)) d s\right\} \mid \\
\leq & \left\{\frac{(m+1) a c}{\delta \Gamma(q+1)}+\frac{(2 m-1) a c+(m+1)(a d+\delta)}{\delta \Gamma(q)}\right\}\|\mu\|_{P C}:=\mathcal{M} .
\end{aligned}
$$

If $t_{1}, t_{2} \in J_{k}$, and $t_{1}<t_{2}, 0 \leq k \leq m$, then

$$
\left|\left(\mathcal{A}_{1} u\right)\left(t_{2}\right)-\left(\mathcal{A}_{1} u\right)\left(t_{1}\right)\right| \leq \int_{t_{1}}^{t_{2}}\left|\left(\mathcal{A}_{1} u\right)^{\prime}(s)\right| d s \leq \mathcal{M}\left(t_{2}-t_{1}\right) .
$$

Thus, $\mathcal{A}_{1}$ is equicontinuous. Using the fact that $f$ maps bounded subsets into relatively compact subsets, it follows that $\mathcal{A}_{1}$ is relatively compact on $B_{r}$. Hence, by the Ascoli-Arzelà theorem, $\mathcal{A}_{1}$ is compact on $B_{r}$. Thus all the assumptions of Lemma 2.5 are satisfied. Hence, by the conclusion of Lemma 2.5, the impulsive fractional boundary value problem (1.1) has at least one solution on $J$.

In the sequel we present an example which illustrates Theorem 3.2.

\section{An example}

Example 4.1 Consider the following boundary value problem:

$$
\left\{\begin{array}{l}
{ }^{c} D_{0+}^{q} u(t)=\frac{\sin t}{(t+3)^{2}} \frac{|u(t)|}{1+(u(t) \mid}, \quad 0 \leq t \leq 1, t \neq \frac{1}{2}, \\
\Delta u\left(\frac{1}{2}\right)=\frac{1}{(t+5)^{2}} \frac{|u(t)|}{16+|u(t)|}, \quad \Delta u^{\prime}\left(\frac{1}{2}\right)=\frac{1}{(t+7)^{2}} \frac{|u(t)|}{25+|u(t)|}, \\
u(0)-u^{\prime}(0)=0.03, \quad u(1)+u^{\prime}(1)=0.06
\end{array}\right.
$$

where $q=\frac{3}{2}, a=1, b=1, c=1, d=1, m=1, \delta=a c+a d+b c=3$. Clearly $\gamma_{1}=\frac{1}{9}, \gamma_{2}=\frac{1}{25}$, $\gamma_{3}=\frac{1}{49}, \gamma_{4}=\frac{1}{25}, \gamma_{5}=\frac{1}{49}, x_{0}=0.03, x_{1}=0.06$.

Moreover, we have

$$
\begin{aligned}
\lambda:= & \frac{m[c(a+b)+\delta]}{\delta} \gamma_{4}+\frac{(2 m-1)[c(a+b)+\delta]+m d(a+b)}{\delta} \gamma_{5} \\
& +\frac{(2 c+d)\left|x_{0}\right|+(a+b)\left|x_{1}\right|}{\delta} \approx 0.1743<1 .
\end{aligned}
$$

Thus, all the assumptions of Theorem 3.2 are satisfied. Hence, by the conclusion of Theorem 3.2, the impulsive fractional boundary value problem (4.1) has at least one solution on $J$.

\section{Competing interests}

The authors declare that they have no competing interests.

\section{Authors' contributions}

All authors contributed equally to the manuscript. All authors read and approved the final manuscript.

\section{Acknowledgements}

This research was supported by the National Natural Science Foundation of China (11161027, 11262009); by the Scientific Research Projects in Colleges and Universities of Gansu Province of China (2013A-043); by the Fundamental Research Funds for the Gansu Universities (2013); by the Fundamental Research Funds for the Gansu Universities (212084); by the Youth Science Foundation of Lanzhou Jiaotong University (2012019); by the National Natural Science Foundation of China (11226132). The authors are thankful to the referees for their careful reading of the manuscript and insightful comments. 


\section{References}

1. Kilbas, AA, Srivastava, HM, Trujillo, JJ: Theory and Applications of Fractional Differential Equations. Elsevier, Amsterdam (2006)

2. Miller, KS, Ross, B: An Introduction to the Fractional Calculus and Differential Equations. Wiley, New York (1993)

3. Oldham, KB, Spanier, J: The Fractional Calculus. Academic Press, New York (1974)

4. Podlubny, I: Fractional Differential Equation. Academic Press, San Diego (1999)

5. Samko, SG, Kilbas, AA, Marichev, Ol: Fractional Integrals and Derivatives, Theory and Applications. Gordon \& Breach, Yverdon (1993)

6. Daftardar-Gejji, V: Positive solutions of a system of non-autonomous nonlinear fractional differential equations. J. Math. Anal. Appl. 302(1), 56-64 (2005)

7. Babakhani, A, Daftardar-Gejji, V: Existence of positive solutions for multi-term non-autonomous fractional differential equations with polynomial coefficients. Electron. J. Differ. Equ. 2006(129), 1-12 (2006)

8. Bai, ZB, Lü, H: Positive solutions for boundary value problem of nonlinear fractional differential equation. J. Math. Anal. Appl. 311(2), 495-505 (2005)

9. Zhou, W-X, Chang, Y-X, Liu, H-Z: Weak solutions for nonlinear fractional differential equations in Banach spaces. Discrete Dyn. Nat. Soc. 2012, Article ID 527969 (2012)

10. Zhou, W-X, Peng, J-G, Chu, Y-D: Multiple positive solutions for nonlinear semipositone fractional differential equations. Discrete Dyn. Nat. Soc. 2012, Article ID 850871 (2012)

11. Zhou, W-X, Chu, Y-D: Existence of solutions for fractional differential equations with multi-point boundary conditions. Commun. Nonlinear Sci. Numer. Simul. 17(3), 1142-1148 (2012)

12. Baĭnov, DD, Simeonov, PS: Systems with Impulsive Effect. Ellis Horwood, Chichester (1989)

13. Benchohra, M, Henderson, J, Ntouyas, S: Impulsive Differential Equations and Inclusions. Hindawi Publishing Corporation, New York (2006)

14. Lakshmikantham, V, Bainov, DD, Simeonov, PS: Theory of Impulsive Differential Equations. Worlds Scientific, Singapore (1989)

15. Samoilenko, AM, Perestyuk, NA: Impulsive Differential Equations. World Scientific, Singapore (1995)

16. Ahmad, B, Sivasundaram, S: Existence results for nonlinear impulsive hybrid boundary value problems involving fractional differential equations. Nonlinear Anal. Hybrid Syst. 3(3): 251-258 (2009)

17. Tian, YS, Bai, Z: Existence results for the three-point impulsive boundary value problem involving fractional differential equations. Comput. Math. Appl. 59(8), 2601-2609 (2010)

18. Zhang, LH, Wang, G: Existence of solutions for nonlinear fractional differential equations with impulses and anti-periodic boundary conditions. Electron. J. Qual. Theory Differ. Equ. 2011(7), 1-11 (2011)

19. Henderson, J, Ouahab, A: Impulsive differential inclusions with fractional order. Comput. Math. Appl. 59(3), 1191-1226 (2010)

20. Agarwal, RP, Benchohra, M, Hamani, S: A survey on existence results for boundary value problems of nonlinear fractional differential equations and inclusions. Acta Appl. Math. 109(3), 973-1033 (2010)

21. Wang, GT, Ahmad, B, Zhang, L: Impulsive anti-periodic boundary value problem for nonlinear differential equations of fractional order. Nonlinear Anal. 74(3), 792-804 (2011)

22. Wang, $\mathrm{XH}$ : Impulsive boundary value problem for nonlinear differential equations of fractional order. Comput. Math Appl. 62(5), 2383-2391 (2011)

23. Wang, GT, Ahmad, B, Zhang, L: Some existence results for impulsive nonlinear fractional differential equations with mixed boundary conditions. Comput. Math. Appl. 62(3), 1389-1397 (2011)

24. Ahmad, B, Nieto, JJ: Existence of solutions for impulsive anti-periodic boundary value problems of fractional order. Taiwan. J. Math. 15(3), 981-993 (2011)

25. Smart, DR: Fixed Point Theorems. Cambridge University Press, Cambridge (1980)

10.1186/1687-1847-2014-12

Cite this article as: Zhou and Liu: Existence of solution to a class of boundary value problem for impulsive fractional differential equations. Advances in Difference Equations 2014, 2014:12

\section{Submit your manuscript to a SpringerOpen ${ }^{\circ}$ journal and benefit from:}

- Convenient online submission

- Rigorous peer review

- Immediate publication on acceptance

- Open access: articles freely available online

- High visibility within the field

- Retaining the copyright to your article 